\author{
Chengpeng Wan \\ Intelligent Transportation Systems Research Center, Wuhan University of Technology, Wuhan, People's Republic \\ of China.E-mail: cpwan@whut.edu.cn
}

Zaili Yang*

Liverpool Logistic Offshore \& Marine Research Institute, Liverpool John Moores University, Liverpool, UK. Email:z.yang@ljmu.ac.uk

Xinping Yan

Intelligent Transportation Systems Research Center, Wuhan University of Technology, Wuhan, People's Republic ofChina.E-mail:xpyan@whut.edu.cn

Di Zhang

Intelligent Transportation Systems Research Center, Wuhan University of Technology, Wuhan, People's Republic of China.E-mail: zhangdi@whut.edu.cn

Eduardo Blanco-Davis

Liverpool Logistic Offshore \& Marine Research Institute, Liverpool John Moores University, Liverpool, UK. Email:e.e.blancodavis@ljmu.ac.uk

Jun Ren

Liverpool Logistic Offshore \& Marine Research Institute, Liverpool John Moores University, Liverpool, UK. Email:j.ren@ljmu.ac.uk

\begin{abstract}
This paper describes a new conceptual framework to facilitate the resilience analysis of maritime container transport networks, by taking into consideration both local risk estimations of container shipping hazards and their global impacts on the overall safety performance of the whole transportation network. In the proposed framework, a series of risk assessment techniques (e.g. Fuzzy and Bayesian networks), are applied to provide a flexible way for transforming risk input information into the evaluations of individual risk factors; and network centrality measures are used to determine the vulnerability of each container shipping route within the network. Furthermore, the evidential reasoning (ER) algorithm is used to aggregate the risk estimations of container shipping operations and importance of container shipping routes in a whole network collectively. Several extreme risk scenarios are set to measure the influence of risk conditions of different container shipping routes on the overall safety performance of the entire network, and the rationality and feasibility of the method are validated by using numerical case studies. The novel framework proposed in this research supports dynamic risk-based resilience analysis of maritime container transport networks. It provides a paradigm shift of resilience analysis by the integration of local level risk analysis of components and global level risk impacts of components to complex networks. It provides the solutions to rationalizing safety resources between the low prioritized hazards of the nodes of high safety impact and the highrisk hazards of the nodes of low safety impact in complex transportation networks in a quantitative manner.
\end{abstract}

Keywords: Container shipping, fuzzy rule-based method, resilience, evidential reasoning, maritime risk and maritime safety.

\section{Introduction}

In the last several decades, increasing volume of international trade, fast development of transportation infrastructure, and ever-changing technology innovation facilitate transport containerization worldwide. However, the growth in globalization and complexity of maritime container transportation systems also brings uncertainties which render high risk for safe and efficient operations. In the past decade, maritime container supply chain risks caused the loss of billions of dollars in the European Union (EU) alone, and the number of accidents and severity of the consequences are growing fast. Safety is one of the issues with great importance in maritime transportation research. However, its foci have been expanded from traditional risk through security, to resilience and sustainability. The involvement of resilience provides a new angle on the risk management of maritime transportation, and thus it attracts considerable interests from both researchers and practitioners across different research domains (Wan et al., 2017).

Regarding the resilience analysis of a transportation network, one most commonly used method is to compare the variation of certain indexes related to the network performance before

\title{
Proceedings of the 29th European Safety and Reliability Conference.
}

Edited by Michael Beer and Enrico Zio

Copyright (c) 2019 European Safety and Reliability Association.

Published by Research Publishing, Singapore.

ISBN: 978-981-11-2724-3; doi:10.3850/978-981-11-2724-3_0213-cd 
and after the removal of some nodes/links (Kim, Chen and Linderman, 2015; Zhen et al., 2016). In the maritime container shipping industry, the removal of a node implies the shutdown of a port, and the removal of a link can be regarded as the changing of service configurations of a shipping company (Viljoen and Joubert, 2016).

In previous studies, disruptions of a transportation network are initially divided into random disruptions and targeted ones. Random disruptions imply the removal of the components (nodes or links) of a transportation network in a random manner, which may be caused by, for example, natural disasters (such as hurricane and earthquake) and unexpected accidents (such as the explosion that temporarily shut down two container terminals at the port of Tianjin, China (Wan et al., 2018). While targeted disruptions (e.g., deliberate attacks) will prioritize the removal of the components following a special strategy such as removing nodes in the order of node degree, link betweenness, and link salience (Viljoen and Joubert, 2016), in order to cause as much damage as possible. However, both ways cannot fully reflect the real situation in practice, which can be regarded as two special cases.

In practice, the occurrence of a disruption usually relates to the risk condition of a transportation network. In other words, a disruption is more likely to occur in the section of a transportation system with a higher risk level. However, it is worth noting that a higher likelihood of disruption does not necessarily indicate a higher impact on the whole transportation system, as the impact of disruption also depends on the specific role it plays in the whole system.

In view of the above debate, this work aims to develop an integrated approach for analyzing the resilience of maritime container transport networks (MCTNs), considering both the risk level of container shipping operations and the relative importance of each shipping route within MCTNs, in order to achieve a paradigm shift on resilience analysis of complex networks such as MCTNs. In order to accomplish the above aim, this work is organized as follows. Section 2 introduces the framework of MCTN resilience analysis, research steps, and major methods used in this work. A numerical case study based on a world-leading container shipping company is conducted to demonstrate the feasibility of the proposed method in Section 3. Finally, this work is concluded in Section 4.

\section{Methodology}

\subsection{Research framework}

The proposed framework for analyzing the resilience of MCTNs consists of three major components, which outline all the necessary steps required, as shown in Fig 1. It can be seen that the methodological view on resilience analysis adopted in this work is originated from two streams. On the one hand, a series of risk models are generated to support the risk factor identification, risk analysis, and assessment of risk levels of container shipping operations, while on the other hand, a network-based approach is developed to calculate the centrality of ports, and measure the relative importance of each shipping routes within the MCTN from a physical structure perspective. The two streams are merged together to quantify the operational performance of MCTNs. In this study, the resilience of MCTNs is measured according to the drop of the operational efficiency of the investigated networks with respect to the increased risk levels of container shipping routes. It is worth noting that although the resilience of a transportation system exists in the whole process when a disruptive event occurs (e.g. pre-disaster, during a disaster, and postdisaster), this study mainly considers the stage during a disruption with focuses on the abilities to withstand and survive in the face of disruptive events.

Three main research steps to support the resilience analysis of MCTNs are listed as follows:

Step 1. Modelling of the topological structure of MCTNs.

Step 2. Quantify the operational performance of MCTNs (this step is further conducted from two aspects, which are:

- $\quad$ Risk assessment of container shipping operations.

- Importance measurement of container shipping routes.

Step 3. Evaluate the resilience of MCTNs through sensitivity analysis. 


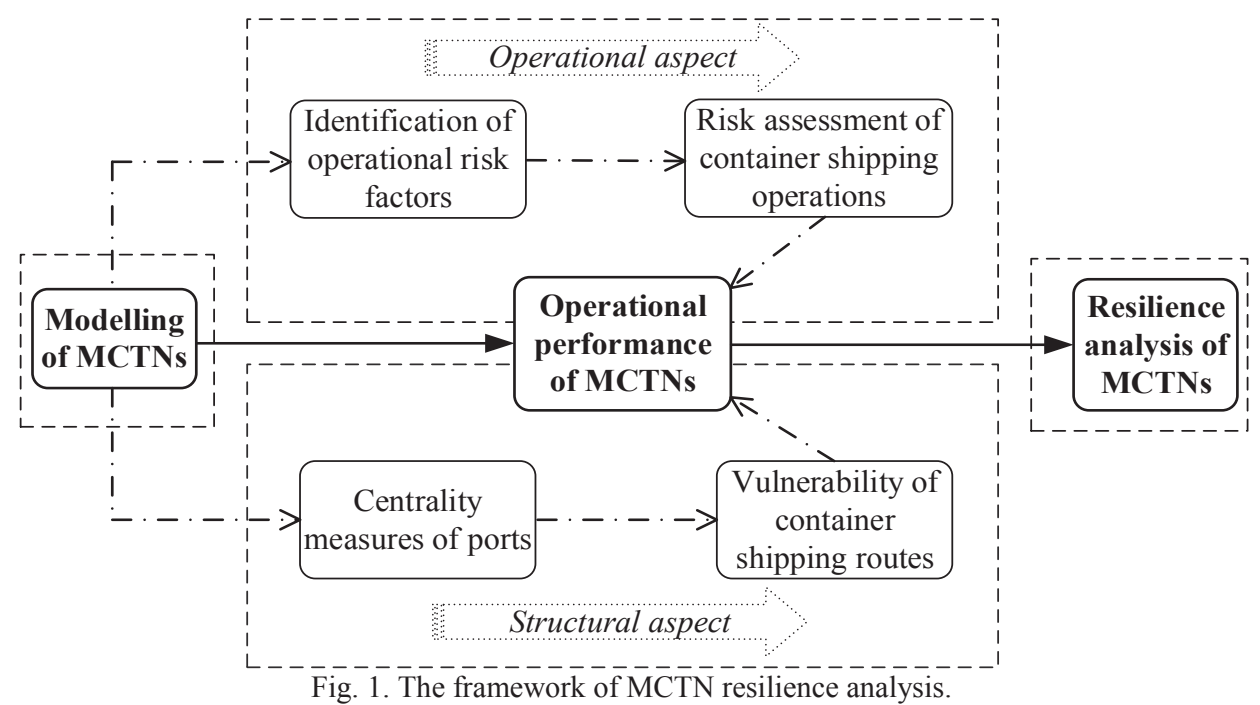

\subsection{Construction of MCTNs}

Due to the fact that a highly interconnected global maritime container transportation system functions with all the features and characteristics of a typical network, it is often abstracted to a graph, in which container ports are viewed as the nodes and liner shipping services as the arcs within the network. Therefore, an MCTN provides an excellent fundamental basis for analyzing its structure by using a complex network approach. In this chapter, an MCTN is abstracted as a connected network $G=(V, E)$ by $V$ and $E$, where $V=\left\{v_{i}: i=1,2, \ldots, n\right\}, n$ is the number of nodes (ports), while $E=\left\{e_{i}: i=1,2, \ldots\right.$, $m\}, m$ is the number of arcs (links between ports). To represent a network, an adjacency matrix $A_{n \times n}$ is created where an element $a_{i j}=1$ when a container liner service exists between port $v_{i}$ and $v_{j}$, and $a_{i j}=0$ otherwise.

\subsection{Measuring the weights of container shipping routes}

\subsubsection{Centralities of ports}

Centrality has already been studied in the maritime transportation sector as an indicator for assessing the relative importance/position of ports as early as in the 1990s (Wang and Cullinane, 2016). In previous studies, the three most widely applied centrality measures in the shipping industry include the degree, closeness, and betweenness centrality. Centrality indices were first developed in social network analysis (Newman, 2010) to study the characteristics of those crucial nodes within a graph so that the most important ones can be identified based on their rankings. In this study, only degree centrality of a port is considered, which is defined as the number of links directly connected to it. Degree centrality of port $v_{i}$ is defined as:

$$
C_{D}(i)=\sum_{j=1}^{n} a_{i j}=k_{i}
$$

\subsubsection{Weights of container shipping routes}

From a network perspective, it was revealed that the weight of a link is strongly correlated with its product degree (Tang and Zhou, 2011), and this assumption has been supported by empirical evidence of real transportation networks such as air networks (Barrat et al., 2004). Without loss of generality, it is supposed that a targeted MCTN is composed of $y$ shipping routes and the shipping route under investigation is composed of $x$ ports. Thus, the importance of each container shipping route within an MCTN can be assigned as (Holme et al., 2002):

$$
L_{a b}=\left(C_{D}(a) C_{D}(b)\right)^{\theta}
$$

where $\theta$ is a tuneable weight parameter (which is usually set as 1$)$, and $C_{D}(a)$ and $C_{D}(b)$ are the degree of ports $a$ and $b(a, b=1,2, \ldots, x ; a \neq b)$ respectively. Supposing that cargos are transported in a sequence from port $a$ to $b$, the overall importance of the entire shipping route can be calculated as the sum of the importance of each section composing this route, expressed as:

$$
W=\sum_{\substack{a=1 \\ b=a+1}}^{x-1} L_{a b}
$$


Therefore, the relative weight of each shipping route within the MCTN can be calculated as:

$$
w(c)=\frac{W(c)}{\sum_{c=1}^{y} W(c)}
$$

where, $w(c)$ is the relative weight of shipping route $c$ in the MCTN.

\subsection{Assessment of operational risks of MCTNs}

\subsubsection{Framework for risk classification in maritime container shipping}

Based on a systematic review of previous studies (e.g., Ho et al., 2015; Wan et al., 2019) and an indepth discussion with domain experts, the framework for operational risk classification is proposed, as shown in Fig. 2. It is a top-down structure framework, which helps to clarify the relationships among different risk sources step by step. It provides the basis for the identification of risk factors. It is composed of three levels (Level I, II, and III), in which the bottom level consists of all the risk factors identified with respect to different aspects of container shipping operations. In this study, altogether 26 operational risk factors are identified such as information delay, charter rates rise, port congestions, and transportation of dangerous goods. Refer to Wan et al., (2019) for more detailed information on the identified risk factors.

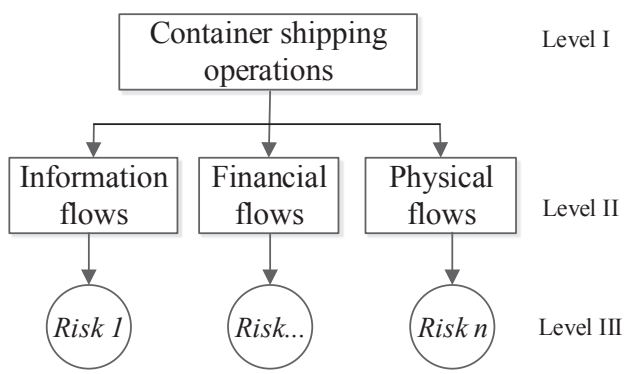

Fig. 2. Classification of operational risk factors in container shipping.

\subsubsection{Assessment of the identified risk factors and the risk condition of shipping routes}

In this study, a fuzzy rule-based method is applied to model the relationship between antecedents (risk parameters such as likelihood and consequence) and consequents (risk status). A belief rule base (BRB) consists of rules which can be used for risk inference, as shown in Eq. (5).

$$
\begin{aligned}
R_{k}: & \operatorname{IF} A_{1}^{k} \text { and } A_{2}^{k} \text { and ...and } A_{M}^{k}, \\
& \operatorname{THEN}\left\{\left(D_{1}, \beta_{1}^{k}\right),\left(D_{2}, \beta_{2}^{k}\right), \ldots,\left(D_{N}, \beta_{N}^{k}\right)\right\} \\
& \left(\sum_{j=1}^{N} \beta_{j}^{k} \leq 1\right)
\end{aligned}
$$

where, $\beta_{j}^{k}(i=1,2, \ldots, N)$ is the degree of belief (DoB) to which $D_{j}$ is believed to be the consequence in the $k$ th packet rule, when the input satisfies the $A^{k}=\left\{A_{1}^{k}, A_{2}^{k}, \ldots, A_{M}^{k}\right\} . N$ is the number of all possible consequents.

In the constructed BRB system, five risk parameters are considered as the antecedent attributes in fuzzy rules (the IF part). They are risk occurrence likelihood $(L)$, visibility $(V)$, consequence severity in terms of time delay/disruption $(C T)$, additional cost $(C C)$, and damage to quality $(C Q)$. Risk status $(R)$ is presented as the consequent attribute (the THEN part). DoBs are assigned to the linguistic variables used to describe the consequent attribute $R$ in the BRB. To facilitate subjective data collection and representation of judgements associated with the five antecedent attributes and conclusion, a set of linguistic variables are defined with three levels (Alyami et al., 2014).

This work adopts a proportion method to rationalize the distribution of DoB. The weight of each risk parameter can be calculated by using the Analytic Hierarchy Process (AHP) method (Saaty, 1980) based on the evaluations of domain experts. Finally, the BRB used in risk assessment of MSCs with $243\left(3^{5}\right)$ rules can be developed. Once all the required data have been collected and prepared, a Bayesian Network (BN) technique is applied to conduct risk inference in order to overcome the drawbacks of traditional rule synthesize methods, including the information loss of the fuzzy Min-Max operation. Using a BN technique, the BRB can be modelled and converted into a converging connection consisting of six nodes: five parent nodes, defined as $N_{L}, N_{V}, N_{C T}, N_{C C}$, and $N_{C Q}$ (Nodes $L, V, C T, C C$ and $C Q$ ); and one child node, defined as $N_{R}$ (Node $R$ ). Having transferred the $\mathrm{BRB}$ into a $\mathrm{BN}$, the rule-based risk inference for the risk assessment is simplified as the calculation of the marginal probability of node $N_{R}$ by using Eq. (6). More details are found from Wan et al., (2019)

$$
\begin{aligned}
& p(R h)=\sum_{i=1}^{3} \sum_{j=1}^{3} \sum_{k=1}^{3} \sum_{l=1}^{3} \sum_{m=1}^{3} p(R h \mid L i, V j, C T k, C C l, C Q m) p(L i) p(V j) p(C T k) p(C C l) p(C Q m) \\
& (h=1,2,3)
\end{aligned}
$$

After each risk factor (in Level III) being assessed, their results under each type of flow (in Level II) can then be combined by using an evidential 
reasoning (ER) approach to obtain the risk condition of information flows, financial flows, and physical flows, respectively. Similarly, the results of the risk condition of different flows will be further synthesized in order to obtain the operational risk condition of the whole shipping route. The ER algorithm will be introduced in Section 2.5.

\subsection{Evaluation of operational performance of MCTNS}

Once the operational risk of shipping routes and their relative weights are obtained, the overall operational performance of the whole MCTN can be calculated by aggregating the risk status of all shipping routes within the MCTN by using the ER approach, which is illustrated as follows.

The set $S(E)=\left\{\left(H_{n}, \beta_{n}\right), n=1, \ldots, N\right\}$ represents a criterion $E$ which is assessed to grade $H_{n}$ with degree of belief $\beta_{n}(n=1, \ldots, N)$. Let $m_{n, i}$ be a basic probability mass representing the degree to which the $i$ th basic criterion $e_{i}$ supports the hypothesis that the criterion $y$ is assessed to the $n$th grade $H_{n}$.

To obtain the combined degrees of belief of all the basic criteria, $E_{I(i)}$ is firstly defined as the subset of the first $i$ basic criteria as follows:

$$
E_{I(i)}=\left\{e_{1}, e_{2}, \ldots, e_{i}\right\}
$$

Let $m_{n, I(i)}$ be a probability mass defined as the degree to which all the $i$ criteria in $E_{I(i)}$ support the hypothesis that $E$ is assessed to the grade $H_{n}$ and let $m_{H, I(i)}$ be the remaining probability mass unassigned to individual grades after all the basic criteria in $E_{I(i)}$ have been assessed.

$$
\begin{gathered}
m_{n, I(1)}=m_{n, 1}, n=1,2, \ldots \ldots, N \\
m_{H, I(1)}=m_{H, 1}
\end{gathered}
$$

By using Eq. (8) and Eq. (9), Eq. (10) can be constructed for $i=1,2, \ldots, L-1$ to obtain the coefficients $m_{n, I(L)}, \bar{m}_{H, I(L)}$ and $\tilde{m}_{H, I(L)}$ (Yang and $\mathrm{Xu}, 2002$ ):

$$
K_{I(i+1)}=\left[1-\sum_{t=1}^{N} \sum_{\substack{j=1 \\ j \neq t}}^{N} m_{t, I(i)} m_{j, i+1}\right]^{-1}
$$

$K_{I(\mathrm{i}+1)}$ is a normalizing factor.

$$
\begin{aligned}
& \left\{H_{n}\right\}: \\
& \quad m_{n, I(i+1)}
\end{aligned}=K_{I(i+1)}\left[m_{n, I(i)} m_{n, i+1}+m_{H, I(i)} m_{n, i+1}+m_{n, I(i)} m_{H, i+1}\right]
$$

$$
\begin{gathered}
n=1,2, \ldots, N \\
\widetilde{m}_{H, I(i+1)}=K_{I(i+1)}\left[\widetilde{m}_{H, I(i)} \widetilde{m}_{H, i+1}+\bar{m}_{H, I(i)} \widetilde{m}_{H, i+1}+\widetilde{m}_{H, I(i)} \bar{m}_{H, i+1}\right] \\
\bar{m}_{H, I(i+1)}=K_{I(i+1)} \bar{m}_{H, I(i)} \bar{m}_{H, i+1}
\end{gathered}
$$

$\{H\}$ :

$$
m_{H, I(i)}=\widetilde{m}_{H, I(i)}+\bar{m}_{H, I(i)}, i=1,2, \ldots, \mathrm{L}-1
$$

The combined DoB of all the basic criteria for the assessment to criterion $E$ is calculated by:

$$
\begin{gathered}
\left\{H_{n}\right\}: \quad \beta_{n}=\frac{m_{n, I(L)}}{1-\bar{m}_{H, I(L)}}, n=1,2, \ldots, N \\
\{H\}: \quad \beta_{H}=\frac{\tilde{m}_{H, I(L)}}{1-\bar{m}_{H, I(L)}}
\end{gathered}
$$

Normally, the synthesized results of the operational risk of the transport network obtained from the aggregating process are presented in the form of linguistic terms with their associated DoBs. This will hinder the comparison of impacts of different shipping routes on the system resilience because linguistic terms (e.g., good, average, and poor) are not sufficient to show the difference between the results. Numerical values, therefore, need to be generated from the obtained distributed results.

Suppose the utility of a performance evaluation grade of maritime supply network $H_{n}$ is denoted by $u\left(H_{n}\right)$ and $u\left(H_{n+1}\right)>u\left(H_{n}\right)$ if $H_{n+1}$ is preferable to $H_{n} . u\left(H_{n}\right)$ can be estimated by the decision maker's preference (Riahi et al., 2012). In this research, there are three evaluation grades associated with the performance of an MCTN, which are Poor, Average, and Good. The utility of each evaluation grade is respectively assigned as follows (Yang, Ng and Wang, 2014):

$$
\begin{aligned}
& u\left(H_{\text {Poor }}\right)=1 \\
& u\left(H_{\text {Avg. }}\right)=10 \\
& u\left(H_{\text {Good }}\right)=100
\end{aligned}
$$

The utility of the performance of maritime supply networks (top-level) $E$ is denoted by $u(E)$, which can be calculated by Eq. (18).

$$
u(E)=\sum_{n=1}^{N} \beta_{n} u\left(H_{n}\right)
$$

\subsection{Resilience analysis of MCTNS}

After the transformation of the linguistic evaluation results into numerical values, sensitivity analysis is required to evaluate 
MCTNs' resilience by measuring the deterioration of the operational performance of the system in the face of different disruptions.

The operational performance of the MCTN under normal situation $\left(O P_{N}\right)$ can be regarded as a baseline, which reflects the risk condition of the MCTN during daily operations. Then, the DoBs associated with the linguistic term High of a shipping route are increased to $100 \%$ to check the performance of the MCTN under extreme risk conditions $\left(O P_{E}\right)$. A resilience index $(R I)$ is proposed according to the ration between $O P_{N}$ and $O P_{E}$, showing the MCTN's ability to resist external disturbance and maintain the service function to a certain level, which can be calculated as:

$$
R I=1-\frac{O P_{N}-O P_{E}}{O P_{N}}
$$

Thus, the higher the value of $R I$, the better an MCTN performs in terms of operational safety.

\section{A numerical study of the Company C}

\subsection{Modelling of the container transport network of the Company $C$}

The sample MCTN chosen for illustration in this study is from a world leading carrier (refer to as "Company C"). The maritime data was sourced from the service information of Company $\mathrm{C}$ published online. The schedule information was collected, and basic information of a specific route includes ports of call (port rotation), time schedule, ship fleet, and ship capacity. A time span of two months is considered in this study as this time period can cover the longest period a voyage may take. By applying the graph of all linkages (GAL) representation, each shipping route can be interpreted as a small network where all ports in the same shipping route are linked together, and the merging of all these individual sub-networks results in the complete network. Based on the information of 123 shipping routes in the dataset, an unweighted and directed network is constructed composing of 212 nodes and 3425 links, as shown in Fig. 3.

\subsection{Operational performance of the Africa MCTN}

In this study, the service on Asia/Africa Trade Lane offered by the Company $\mathrm{C}$ will be taken as a case study. The regional MCTN in Africa is composed of five major shipping routes, namely, FAX route, ASA route, AEF route, ASEA route, and FWAS route. There are altogether 36 container ships serving 41 port pairs, covering the eastern, western, and southern Africa.

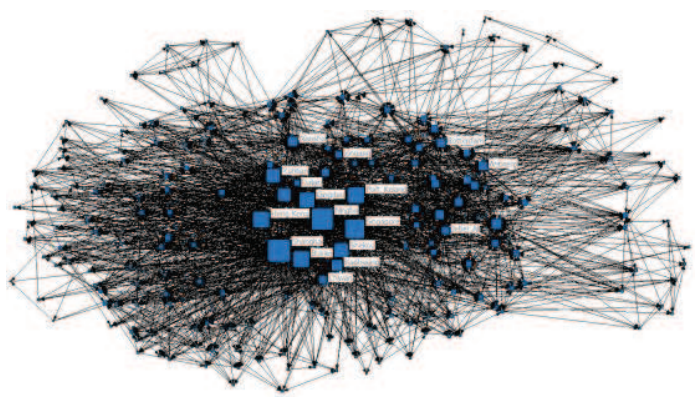

Fig. 3. Graph of the sample network

Source: realized by the author based on the service data from Company $\mathrm{C}$ using the NetDraw software.

First of all, the degree centrality of all ports can be obtained based on Eq. (1), and the top three ports in terms of degree centrality are Port of Shanghai (241), Port of Singapore (230), and Port of Ningbo (180). Based on that, the weights of each shipping routes can be obtained by using Eqs. (2), (3), and (4), as summarized in Table 1.

Next step is to assess the risk condition of each shipping route. Normally, a questionnaire survey can be conducted to collect risk assessment information on the 26 identified operational risk factors with respect to each shipping route, and the feedback received from different experts will be combined using a weighted average method. The estimations of operational risk factors will be synthesized to obtain the risk condition of the shipping route. Since this work mainly focuses on the resilience analysis of MCTNs, here, it is assumed that the risk conditions of operations of shipping routes under normal situations are as follows:

- $\quad$ FAX: $\{($ Low, $10 \%),($ Medium, $20 \%)$, (High, $70 \%)\}$

- $\quad$ ASA: $\{($ Low, $70 \%)$, (Medium, 20\%), (High, 10\%) $\}$

- $\quad$ AEF: $\{($ Low, 33\%), (Medium, 33\%), (High, 34\%)\}

- $\quad$ ASEA: $\{($ Low, $0 \%)$, (Medium, 50\%), (High, 50\%)\}

- $\quad$ FWAS: $\{($ Low, 50\%), (Medium, 50\%), (High, 0\%)\}

Taking the risk condition of FAX as an example, it indicates that its operational risk level is Low with a $10 \%$ DoB, Medium with a $20 \%$ DoB and High with a $70 \%$ DoB. The $O P_{N}$ of the MCTN can be calculated by using Eqs from (7) to (16), which is estimated to be $\{($ Poor, 20.0\%), (Average, $35.5 \%)$, (Good, 44.5\%) $\}$. Its expected value can be calculated based on Eq. (17) and (18), which is:

$u\left(O P_{N}\right)=\sum_{n=1}^{3} \beta_{n} u\left(H_{n}\right)=0.2 \times 1+0.355 \times 10+0.445 \times 100=48.250$ 


\subsection{Resilience analysis of the Africa MCTN}

In order to analyze the resilience of the Africa MCTN, different extreme scenes are set in the sensitivity analysis by increasing the DoB belonging to the linguistic to $100 \%$ with respect to different combinations of shipping routes. Explanations of different scenarios along with the excepted utilities are summarized in Table 2. According to Eq. (19), the RI value under different risk situations can be obtained. Taking FAX as an illustration, the resilience index with respect to its disruption can be calculated as:

$$
R I_{F A X}=1-\frac{O P_{N}-O P_{E-F A X}}{O P_{N}}=1-\frac{48.250-45.721}{48.250}=94.76 \%
$$

It shows that $94.76 \%$ of the performance of the Africa MCTN remains in terms of the operational safety after the disruption of FAX route. Similarly, the RI value of other routes can be calculated as $R I_{\mathrm{ASA}}=58.55 \%, R I_{\mathrm{AEF}}=96.42 \%$, $R I_{\mathrm{ASEA}}=99.93 \%$, and $R I_{\mathrm{FWAS}}=40.91 \%$, respectively.

Table 1. Ports of call and relative weights of different shipping routes in the Africa region

\begin{tabular}{|c|c|c|}
\hline $\begin{array}{l}\text { Shipping } \\
\text { Route }\end{array}$ & $\begin{array}{l}\text { Relative } \\
\text { weight }\end{array}$ & Ports of call \\
\hline FAX & 0.242 & Shanghai-Ningbo-Keelung-Singapore-Durban-Singapore-Shanghai \\
\hline ASA & 0.254 & $\begin{array}{l}\text { Kaohsiung-Xiamen-Hong Kong-Shekou-Singapore-Port Kelang-Durban-Cape Town } \\
\text {-Port Kelang-Singapore-Kaohsiung }\end{array}$ \\
\hline $\mathrm{AEF}$ & 0.055 & Port-Kelang-Singapore-Colombo-Mombasa-Colombo-Port Kelang \\
\hline ASEA & 0.054 & Singapore-Port Kelang-Colombo-Dar es Salaam-Colombo-Singapore \\
\hline FWAS & 0.395 & $\begin{array}{l}\text { Shanghai-Ningbo-Hong Kong-Nansha-Singapore-Apapa-Tema-Lome-Abidjan } \\
\text {-Singapore-Shanghai }\end{array}$ \\
\hline
\end{tabular}

Table 2. Operational performance of the Africa MCTN under different scenarios

\begin{tabular}{llllll}
\hline \multicolumn{2}{l}{ Normal situation } & $\boldsymbol{O P}_{N}$ & \multicolumn{2}{l}{ Extreme scenario 1 } & $\boldsymbol{O P}_{\text {E-FAX }}$ \\
\hline FAX & $(0.1,0.2,0.7)$ & & FAX & $(0,0,1)$ & \\
ASA & $(0.7,0.2,0.1)$ & & ASA & $(0.7,0.2,0.1)$ & \\
AEF & $(0.33,0.33,0.34)$ & 48.250 & AEF & $(0.33,0.33,0.34)$ & 45.721 \\
ASEA & $(0,0.5,0.5)$ & & ASEA & $(0,0.5,0.5)$ & \\
FWAS & $(0.5,0.5,0)$ & & FWAS & $(0.5,0.5,0)$ & \\
\hline Extreme scenario 2 & $\boldsymbol{O P}_{E-A S A}$ & Extreme scenario 3 & $\boldsymbol{O P}_{\text {E-AEF }}$ \\
\hline FAX & $(0.1,0.2,0.7)$ & & FAX & $(0.1,0.2,0.7)$ & \\
ASA & $(0,0,1)$ & & ASA & $(0.7,0.2,0.1)$ & \multirow{2}{*}{ A.522 } \\
AEF & $(0.33,0.33,0.34)$ & 28.252 & AEF & $(0,0,1)$ & \\
ASEA & $(0,0.5,0.5)$ & & ASEA & $(0,0.5,0.5)$ & \\
FWAS & $(0.5,0.5,0)$ & & FWAS & $(0.5,0.5,0)$ & \\
\hline Extreme scenario 4 & $\boldsymbol{O P}_{E-A S E A}$ & Extreme scenario 5 & $\boldsymbol{O P}_{\text {E-FWAS }}$ \\
\hline FAX & $(0.1,0.2,0.7)$ & & FAX & $(0.1,0.2,0.7)$ & \\
ASA & $(0.7,0.2,0.1)$ & & ASA & $(0.7,0.2,0.1)$ & \\
AEF & $(0.33,0.33,0.34)$ & 48.214 & AEF & $(0.33,0.33,0.34)$ & 19.738 \\
ASEA & $(0,0,1)$ & & ASEA & $(0,0.5,0.5)$ & \\
FWAS & $(0.5,0.5,0)$ & & FWAS & $(0,0,1)$ & \\
\hline
\end{tabular}

It can be seen that the broken of different shipping routes will lead to different degrees of deterioration of the MCTN performance, among which the shutdown of FWAS route influences the most. However, it is also worth noting that the whole network still remains an operational function of $40.91 \%$ even under the most severe risk scenario, showing its ability to survive in the face of external disturbance. Regarding the occurrence of extreme situations on these shipping routes in succession, the operational performance shows a trend of continuous decline, 


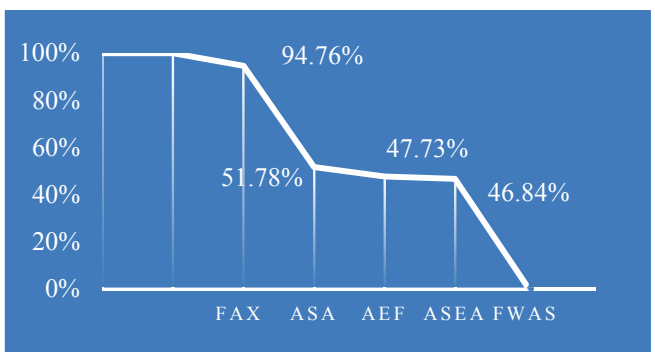

Fig. 4. Remaining operational performance after the disruption of each shipping routes one by one as shown in Fig. 4. Finally, the whole MCTN almost loses its service functions when all the shipping routes are at the highest risk level.

\section{Conclusions}

This work proposes a novel framework incorporating different advanced risk modelling and assessment techniques in a complementary way, to achieve the risk based resilience analysis of MCTN from a systematical perspective. Although the case study of the proposed model in this study focuses on the operational aspects, leaving the other risk sources such as managerial, natural environment, and political issues to be addressed in future work, the proposed method, for the first time, reveals the novel and innovative idea on comprehensive risk management of maritime supply chains by the integration of local component level operational risks and systematic level safety impacts. The risk-based resilience analysis can provide significant insights for the development of appropriate risk control options to eliminate or mitigate the risk factors in global container logistics systems, and to enhance their robustness and efficiency. It is also noteworthy that the proposed framework provides a standard, generic method for the evaluation of MCSC performance. Although it is applied and demonstrated in a case study of the container shipping industry, it has the potential and flexibility to be tailored to meet the needs of the application in other complex networks such as road and rail transportation.

\section{Acknowledgement}

This research was supported by the EU Marie Curie projects RESET (reference no. 730888) and GOLF (reference no. GOLF-777742).

\section{References}

Alyami, H., Lee, P., Yang, Z., et al. (2014) An advanced risk analysis approach for container port safety evaluation. Maritime Policy \& Management, 41(7), 634-650.

Barrat, A., Barthélemy, M., Pastor-Satorras, R. and Vespignani, A. (2004) The architecture of complex weighted networks. Proceedings of the National Academy of Sciences of the United States of America, 101(11), 3747-52.
Ho, W., Zheng, T., Yildiz, H. and Talluri, S (2015) Supply chain risk management: A literature review. International Journal of Production Research, 53(16), 5031-5069.

Holme, P., Kim, B. J., Yoon, C. N. and Han, S. K. (2002) Attack vulnerability of complex networks. Physical Review E Statistical Nonlinear \& Soft Matter Physics, 65(2), 056109.

Kim, Y., Chen, Y. S. and Linderman, K. (2015) Supply network disruption and resilience: a network structural perspective. Journal of Operations Management, 33-34, 43-59.

Newman, M. (2010) Networks: An Introduction. Oxford: Oxford University Press, Inc.

Satty, T.L. (1980) The Analytic Hierarchy Process. McGraw Hill International, New York, USA.

Tang, M. and Zhou, T. (2011) Efficient routing strategies in scale-free networks with limited bandwidth. Physical Review E Statistical Nonlinear \& Soft Matter Physics, 84(2), 026116.

Viljoen, N.M. and Joubert, J. (2016) The vulnerability of the global container transport network to targeted link disruption. Physica A Statistical Mechanics \& Its Applications, 462, 396-409.

Wan, C., Yan, X., Zhang, D., Qu, Z. and Yang, Z. (2019). An advanced fuzzy belief rule-based Bayesian network approach for maritime supply chain risk analysis. Transportation Research Part E Logistics and Transportation Review, in press.

Wan, C., Yan, X., Zhang, D. and Yang, Z. (2018). A novel model for the quantitative evaluation of green port development - a case study of major ports in China. Transportation Research Part D Transport and Environment, 61(B), 431-443.

Wan, C., Yan, X., Zhang, D. and Yang Z. (2019). Analysis of risk factors influencing the safety of maritime container supply chains. International Journal of Shipping and Transport Logistics, in press.

Wan, C., Yang, Z., Zhang, D., Yan, X. and Fan, S. (2018). Resilience in transportation systems: a systematic review and future directions. Transport Reviews, 38(4), 479-498.

Wang, Y. and Cullinane, K. (2016). Determinants of port centrality in maritime container transportation. Transportation Research Part E Logistics \& Transportation Review, 95, 326-340.

Yang, J. B. and Xu, D. L. (2002). On the evidential reasoning algorithm for multiple attribute decision analysis under uncertainty. IEEE Transactions on Systems Man \& Cybernetics: Part A Systems \& Humans, 32(3), 289-304.

Yang, Z., Ng, A. K. Y. and Wang, J. (2014). A new risk quantification approach in port facility security assessment. Transportation Research Part A Policy and Practice, 59(1), 72-90.

Zhen, X., Li, Y., Cai, G. and Shi, D. (2016). Transportation disruption risk management: business interruption insurance and backup transportation. Transportation Research Part E Logistics and Transportation Review, 90, 51-68. 